\title{
Overcoming the chromatin barrier to end resection
}

\author{
Cell Research (2013) 23:317-319. doi:10.1038/cr.2012.148; published online 13 November 2012
}

\begin{abstract}
Repair of double-strand breaks by homologous recombination requires 5' -3 ' resection of the DNA ends to create $3^{\prime}$ ' single-stranded DNA tails. While much progress has been made in identifying the proteins that directly participate in end resection, how this process occurs in the context of chromatin is not well understood. Two papers in Nature report that Fun30, a poorly characterized member of the Swi2/Snf2 family of chromatin remodelers, plays a role in end processing by facilitating the Exo1 and Sgs1-Dna2 resection pathways.
\end{abstract}

DNA double-strand breaks (DSBs) are highly cytotoxic lesions that must be repaired appropriately to prevent the formation of deleterious chromosome rearrangements associated with tumorigenesis. Cells use two major pathways to repair DSBs: homologous recombination (HR) and non-homologous end joining (NHEJ). Repair by HR requires a homologous donor duplex and is considered a high-fidelity process, whereas the homology-independent end joining pathway involves re-ligation of the broken ends and is more error prone. A critical determinant of repair pathway choice that commits cells to HR instead of NHEJ is the initiation of $5^{\prime}-3^{\prime}$ resection of the DSB ends [1]. Genetic studies in Saccharomyces cerevisiae identified the Mre11-Rad50-Xrs2 (Xrs2 is known as NBS1 or NBN in human) complex and Sae2 as key factors in the initiation of resection by removing oligonucleotides from the $5^{\prime}$ ends to form short 3' single-stranded DNA (ssDNA) tails, while the Exo1 exonuclease or the Sgs1 helicase functioning with the Dna2 endonuclease promote extensive resection in a redundant manner [2-4]. Resection has been reconstituted in vitro with these proteins [5-7]; however, additional factors must be present in vivo to facilitate resection in the context of chromatin.

Previous studies have suggested that cells use both histone modifying and remodeling complexes to relax chromatin and hence facilitate DNA repair. After sensing of a DSB by the MRX complex, the Tell kinase (ATM in human) is activated and phosphorylates histone $\mathrm{H} 2 \mathrm{~A}$ over a large region from the break site, followed by histone acetylation that unwinds chromatin and facilitates the recruitment of remodeling complexes [8]. ATP-dependent remodelers are large multi-subunit complexes that couple ATP hydrolysis to movement of histones or nucleosomes, including exchange or incorporation of core histones or histone variants, eviction of histones or nucleosomes, and repositioning or sliding of nucleosomes, thereby modifying chromatin structure [9]. Several chromatin remodeling complexes, including INO80, SWR1, SWI/SNF and RSC in budding yeast, have been reported to participate in the DNA damage response. It has been proposed that INO80 facilitates the eviction or sliding of nucleosomes in the immediate vicinity of the break site to allow $5^{\prime}-3^{\prime}$ strand resection [10]. The SWR1 complex was suggested to exchange modified histones after repair, while SWI/SNF may facilitate clearing of nucleosomes surrounding the break site prior to Rad51-mediated strand invasion [8]. RSC is believed to affect resection initiation by facilitating Mre11 binding [9].
Two recent studies report that Fun30, a poorly characterized ATP-dependent chromatin remodeler, promotes DNA end resection in Saccharomyces cerevisiae. Both groups identified Fun30 by genome-wide screens for mutants with increased frequencies of recombination between a transformed linear DNA fragment and homologous chromosomal sequences. Chen et al. [11] found that deletion of FUN30 caused increased gene targeting, while Costelloe et al. [12] found higher break-induced replication and gap repair efficiencies in the fun304 mutant, properties shared by the resection mutants $\operatorname{sgs} 1 \Delta$ and exo1 14 . Using several different assays to monitor the formation of ssDNA at endonuclease-induced DSBs, both groups demonstrated that Fun30 promotes extensive resection by Exo1dependent and Sgs1-Dna2-dependent pathways (Figure 1). Indeed, both the fun $30 \Delta \operatorname{sgs} 1 \Delta$ and fun304exo14 double mutants exhibited a more severe resection defect than any of the three single mutants $[11,12]$.

The effect of Fun 30 on end resection could be direct or indirect. Evidence in support of a direct role was provided by both studies showing that Fun 30 localized to DSBs and along the DNA from the break site with similar kinetics as Sgs1, Dna2 and Exo1 [11, 12]. Furthermore, Chen et al. [11] showed that Fun 30 co-immunoprecipitates with RPA, Dna2 and Exo1, and enrichment of these resection factors at DSBs was reduced in the fun $30 \Delta$ mutant. In addition, overexpression of Exo1 in the fun 304 strain was able to rescue both resection and resistance to the topoisomerase I inhibitor camptothecin (CPT) [12]. 
DSB

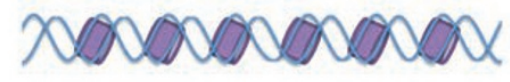

200000000000 Sensing DSB

Extensive resection

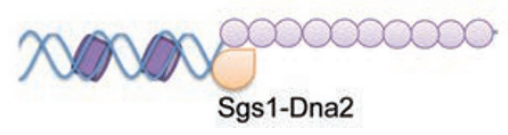

Sgs1-Dna2

Figure 1 The involvement of chromatin remodelers in DSB end resection. Resection initiation is stimulated by RSC and to a lesser extent by INO80. Fun30 works with RPA, Dna2 and Exo1 to promote extensive resection, possibly through overcoming the resection barrier formed by Rad9-bound chromatin.

These data suggest a direct involvement of Fun30 in long-range end resection, possibly through its interaction with extensive resection factors. It remains to be determined whether Fun30 directly recruits the resection machinery, or Fun30-mediated chromatin remodeling facilitates access of resection proteins to ssDNA.

Importantly, the ATPase activity of Fun30, which is essential for its chromatin remodeling activity [13], was found to be required for efficient resection and resistance to CPT [11, 12], indicating a correlation between the two processes. Chen et al. [11] reported impaired recruitment of Fun30 to DSBs in the resection-defective mre11 14 and sgs $1 \Delta$ exold mutants, suggesting that Fun30-mediated chromatin remodeling is coupled with resection. Consistently, ChIP analysis of histone $\mathrm{H} 3$ and $\mathrm{H} 2 \mathrm{~B}$ occupancy around an endonucleaseinduced DSB showed the same trend as resection in wild-type, fun 304 and sgs $1 \Delta$ exolA cells $[11,12]$. Further studies are needed to investigate which one is the causal process, histone eviction or resection. Although histone loss appeared to be slower in fun 304 and sgs1 14exo14 than in wild-type cells, it could be due to impaired long-range resection. Thus Fun30 does not seem to function via evicting histones. It remains to be determined how Fun30 remodels chromatin structure to facilitate resection.

Costelloe et al. [12] extended their findings to human cells by showing that SMARCAD1, the potential human counterpart of Fun30, participates in end resection. SMARCAD1 co-localizes with $\gamma \mathrm{H} 2 \mathrm{AX}$ to DSBs and the pattern of its accumulation at DSBs is similar to that of Exo1. Knockdown of SMARCAD1 caused a dramatic reduction in ionizing radiation-induced ssDNA formation and RPA loading, indicating impaired resection. Accordingly, cells depleted of SMARCAD1 displayed hypersensitivity to genotoxic drugs and reduced HR.

Previous studies suggested that the ATP-dependent nucleosome remodeling complexes, INO80, RSC and SWR, affect resection. Chen et al. [11] sought to characterize the genetic interaction of these remodelers with Fun30 in promoting resection. Of all the single mutants, fun 304 showed the strongest phenotype. Deleting components of the INO80 or RSC complexes together with Fun30 further delayed resection and elimination of all three remodeling factors resulted in a severe resection defect, indicating that Fun30 is the primary activity with RSC and INO80 playing redundant roles (Figure 1).

A further clue to the mechanism by which Fun 30 promotes resection was revealed by its genetic interaction with Rad9, a histone-bound checkpoint mediator known to inhibit resection. Surprisingly, rad 94 was able to suppress the resection defect of fun 304 [11], suggesting that Fun30 is able to overcome the barrier to resection by Rad9-bound chromatin. Consistent with this hypothesis, elimination of Fun30 led to more Rad9 accumulation at DSBs. Understanding how Fun30 is recruited to DSBs and how it recruits other factors is likely to shed some light on its role in resection. $\gamma \mathrm{H} 2 \mathrm{~A}$ is required for the recruitment of INO80 and SWR [8], while recruitment of RSC absolutely requires Mre11 and partially depends on yKu70 [14]. It will be interesting to know whether Fun30 directly interacts with $\gamma \mathrm{H} 2 \mathrm{~A}$ and $\mathrm{Rad} 9$, since $\operatorname{Rad} 9$ is partly recruited by $\gamma \mathrm{H} 2 \mathrm{~A}$. According to Chen et al., recruitment of Fun30 and extensive resection factors to DSBs occurs in a mutually dependent manner. One possible explanation for this paradox is that some initial binding of extensive resection factors facilitates Fun30 localization, which in turn remodels the chromatin and makes it more accessible for more resection factors, forming a positive feedback loop.

As more ATP-dependent chromatin 
remodelers with roles in DNA DSB repair are identified, more questions regarding their apparent functional redundancy are raised. Why do cells need so many complexes to remodel chromatin during DSB repair? How is their sequential recruitment to DSBs regulated and does the apparent redundancy reflect the ordered recruitment? Is recruitment of the early- and late-acting chromatin remodelers coordinated? Do the chromatin remodelers that facilitate end resection participate in later steps of repair, such as invasion of the donor locus by the Rad51-ssDNA complex and resolution of recombination intermediates?
Huan Chen ${ }^{1}$,

Lorraine S Symington ${ }^{1}$

${ }^{1}$ Department of Microbiology \& Immunology, Columbia University Medical Center, New York, NY 10032, USA

Correspondence: Lorraine S Symington

E-mail: 1ss5@columbia.edu

\section{References}

1 Symington LS, Gautier J. Annu Rev Genet 2011; 45:247-271.

2 Gravel S, Chapman JR, Magill C, et al. Genes Dev 2008; 22:2767-2772.

3 Mimitou EP, Symington LS. Nature 2008; 455:770-774.

4 Zhu Z, Chung WH, Shim EY, et al. Cell 2008; 134:981-994.
5 Cejka P, Cannavo E, Polaczek P, et al. $\mathrm{Na}$ ture 2010; 467:112-116.

6 Nicolette ML, Lee K, Guo Z, et al. Nat Struct Mol Biol 2010; 17:1478-1485.

7 Niu H, Chung WH, Zhu Z, et al. Nature 2010; 467:108-111.

8 Loizou JI, Murr R, Finkbeiner MG, et al. Cell Cycle 2006; 5:696-701.

9 Chambers AL, Downs JA. Prog Mol Biol Transl Sci 2012; 110:229-261.

10 van Attikum H, Fritsch O, Hohn B, et al. Cell 2004; 119:777-788.

11 Chen X, Cui D, Papusha A, et al. Nature 2012; 489:576-580.

12 Costelloe $\mathrm{T}$, Louge R, Tomimatsu N, et al. Nature 2012; 489:581-584.

13 Awad S, Ryan D, Prochasson P, et al. J Biol Chem 2010; 285:9477-9484.

14 Shim EY, Ma JL, Oum JH, et al. Mol Cell Biol 2005; 25:3934-3944. 